Special issue of the 3rd International Conference on Computational and Experimental Science and Engineering (ICCESEN 2016)

\title{
Null Quaternionic Cartan Helices in $\mathbb{R}_{\mathrm{v}}^{3}$
}

\author{
A. TUnA AKSOY ${ }^{a, *}$ AND A.C. ÇÖKEN ${ }^{b}$ \\ ${ }^{a}$ Süleyman Demirel University, Department of Mathematics, Isparta, Turkey \\ ${ }^{b}$ Akdeniz University, Department of Mathematics, Antalya, Turkey
}

\begin{abstract}
In this study, we define null quaternionic Bertrand curves in $\mathbb{R}_{\mathrm{v}}^{3}$ for a null quaternionic curve, which has a single non-zero, constant Cartan curvature $\tau$. We also prove that if a null quaternionic curve with non-zero curvatures in $\mathbb{R}_{\mathrm{v}}^{3}$ is a null quaternionic Bertrand curve, then it is a null quaternionic helix.
\end{abstract}

DOI: 10.12693/APhysPolA.132.896

PACS/topics: $02.40 . \mathrm{k}, 02.40 . \mathrm{Hw}$

\section{Introduction}

In a semi-Riemannian manifold, there exist three families of curves, these are, spacelike, timelike and null (lightlike) curves, according to their causal characters. Null curves have different properties from the spacelike and timelike curves and the results of null curve theory are not analogues to Riemannian case. Due to the importance of the null curves in mathematical physics, many mathematicians have studied the differential geometry of null curves.

Bonnor describes geometry of null curves in Minkowski spacetime and uses Cartan frame to study the behaviors of a null curve [2]. Duggal and Bejancu obtained the Frenet equations and proved certain theorems of existence and uniqueness for null curves in semi-Riemannian manifolds [4]. A. Ferrandez, A. Gimenez, and P. Lucas show that a null Frenet curve, parametrized by the pseudoarc parameter, is a null helix, if its lightlike curvature is constant [6]. Then, Honda and Inoguchi [7] have studied deformations of Cartan-framed null curves in the Minkowski 3-space, which preserve the torsion. Later, Inoguchi and Lee have studied some characterizations of null helices in the Minkowski 3-space [8]. Recently, Duggal and Jin [5] have given null Cartan helices for null curves in $\mathbb{R}_{1}^{3}$.

The quaternion was introduced by Hamilton. His initial attempt to generalize the complex numbers by introducing a three-dimensional object had failed in the sense that the algebra he constructed for these threedimensional objects did not have the desired properties. In 1843 Hamilton discovered that the appropriate generalization is one in which the scalar (real) axis is left unchanged, whereas the vector(imaginary) axis is supplemented by adding two further vectors axes.

The theory of Frenet frames for a quaternionic curve was studied and developed by several researchers in this field $[1,3]$. In [1], Bharathi and Nagaraj have defined Serret-Frenet formulas for a quaternionic curve in $\mathrm{E}^{3}$

*corresponding author; e-mail: abidebytr@yahoo.com and $\mathrm{E}^{4}$, and then Çöken and Tuna have studied SerretFrenet formulas for quaternionic curves and quaternionic inclined curves in Semi-Euclidean spaces [3]. Moreover, they studied null quaternionic curves in semi Euclidean 3spaces $\mathbb{R}_{\mathrm{v}}^{3}$ and gave the Frenet formula for null quaternionic curves [10, 11]. Then, Keçilioğlu and İlarslan defined quaternionic Bertrand curves in Euclidean 4-spaces [9].

The main goal of this paper is to define null quaternionic Bertrand curves in the three-dimensional semi Euclidean spaces. Here, by using the similar idea of Duggal and Jin [5], we show that a null quaternionic curve is a Bertrand curve, if and only if, it has non-zero constant second Frenet curvature. In the particular case, where null quaternionic curves are parametrized by the pseudoarc length parameter, null quaternionic helices are the only null quaternionic Bertrand curves. We prove that the distance between null quaternionic Bertrand curves is a constant. Null quaternionic Bertrand curves are characterizated with curvatures.

\section{Preliminaries}

Let $Q_{H}$ denote a four dimensional vector space over the field $H$ of characteristic grater than 2 . Let $e_{i}(1 \leq$ $i \leq 4)$ denote a basis for the vector space. Let the rule of multiplication on $Q_{H}$ be defined on $e_{i}(1 \leq i \leq 4)$ and extended to the entire vector space by distributivity as follows.

The set of the semi real quaternions is defined by $Q_{\mathrm{v}}=\left\{q \mid q=a e_{1}+b e_{2}+c e_{3}+d ; \quad a, b, c, d \in \mathbb{R}, e_{1}, e_{2}, e_{3}\right.$ $\left.\epsilon \mathbb{R}_{\mathrm{v}(\mathrm{v}=1,2)}^{3}, h_{\mathrm{v}}\left(e_{i}, e_{i}\right)=\varepsilon\left(e_{i}\right), 1 \leq i \leq 3\right\}$, where $e_{i} \times$ $e_{i}=-\varepsilon\left(e_{i}\right), 1 \leq i \leq 3, e_{i} \times e_{j}=\varepsilon\left(e_{i}\right) \varepsilon\left(e_{j}\right) e_{k}$ in $\mathbb{R}_{1}^{3}$, $e_{i} \times e_{j}=-\varepsilon\left(e_{i}\right) \varepsilon\left(e_{j}\right) e_{k}$ in $\mathbb{R}_{2}^{3}$ and $(i j k)$ is an even permutation of (123).

The multiplication of two semi real quaternions $p$ and $q$ is defined by

$$
p \times q=S_{p} S_{q}+S_{p} V_{q}+S_{q} V_{p}+h\left(V_{p} V_{q}\right)+V_{p} \Lambda V_{q},
$$

for every $p, q \in Q_{\mathrm{v}}$, where we have used the inner and cross products in semi-Euclidean space $\mathbb{R}_{\mathrm{v}}^{3}$. For a semi real quaternion $q=a e_{1}+b e_{2}+c e_{3}+d \epsilon Q_{\mathrm{v}}$ the conjugate $\alpha q$ of $q$ is defined by

$$
\alpha q=-a e_{1}-b e_{2}-c e_{3}+d .
$$


In this section, we recall the notion of null quaternionic curve in $\mathbb{R}_{\mathrm{v}}^{3}$. Let $C: I \subset \mathbb{R} \rightarrow \mathbb{R}_{\mathrm{v}}^{3}$ be a null quaternionic curve in 3 -dimensional semi Euclidean space. $\left(\mathbb{R}_{\mathrm{v}}^{3}, h_{\mathrm{v}}\right)$ is defined by a semi quaternionic metric

$$
h_{1}(p, q)=\frac{1}{2}[\varepsilon(p) \varepsilon(\alpha q)(p \times \alpha q)+\varepsilon(q) \varepsilon(\alpha p)(q \times \alpha p)]
$$

for $\mathbb{R}_{1}^{3}$ and

$$
h_{2}(p, q)=\frac{1}{2}[-\varepsilon(p) \varepsilon(\alpha q)(p \times \alpha q)-\varepsilon(q) \varepsilon(\alpha p)(q \times \alpha p)]
$$

for $\mathbb{R}_{2}^{3}$. And then, the norm of semi real quaternion $q$ is denoted by

$$
\|q\|^{2}=\left|h_{\mathrm{v}}(q, q)\right|=\left|a^{2} \varepsilon\left(e_{1}\right)+b^{2} \varepsilon\left(e_{2}\right)+c^{2} \varepsilon\left(e_{3}\right)+d^{2}\right|,
$$

for $\mathrm{v}=\{1,2\}$. The concept of a spatial quaternion will be made use throughout our work. $q$ is called a spatial quaternion whenever $q+\alpha q=0$. It is a temporal quaternion whenever $q-\alpha q=0$ [1, 3, 9-11].

\section{Null quaternionic Cartan frames in $\mathbb{R}_{\mathbf{v}}^{3}$}

Next we recall some standard facts concerning null quaternionic curves in the Minkowski 3-space. Let $h$, denote the semi quaternionic metric on $\mathbb{R}_{\mathrm{v}}^{3}$. A curve $C(s)$ in $\mathbb{R}_{\mathrm{v}}^{3}$ is a null quaternionic curve if $h\left(C^{\prime}(s), C^{\prime}(s)\right)=0$ and $C^{\prime}(s) \neq 0$ for all $s$. We note, that a null quaternionic curve $C(s)$ in $\mathbb{R}_{\mathrm{v}}^{3}$ satisfies $h\left(C^{\prime \prime}(s), C^{\prime \prime}(s)\right) \neq 0$. We say that a null quaternionic curve $C(s)$ in $\mathbb{R}_{\mathrm{v}}^{3}$ is parametrized by the pseudo-arc if $h\left(C^{\prime \prime}(s), C^{\prime \prime}(s)\right)=\mp 1$.

Let $C(s)=C_{1}(s) e_{1}+C_{2}(s) e_{2}+C_{3}(s) e_{3}$ be a null quaternionic curve in $\mathbb{R}_{\mathrm{v}}^{3}$. The three-dimensional semi Euclidean space $\mathbb{R}_{\mathrm{v}}^{3}$ is identified with the space of null spatial quaternions $\left\{C \in Q_{\mathbb{R}_{\mathrm{v}}^{3}} \mid C+\alpha C=0\right\}$ in an obvious manner. $C: I \subset \mathbb{R} \rightarrow Q_{\mathbb{R}_{\mathrm{v}}^{3}}, s \rightarrow C(s)=\sum_{i=1}^{3} C_{i}(s) e_{i}$, $1 \leq i \leq 3$. Let $\{l, n, u\}$ be the Frenet trihedron of the differentiable semi-Euclidean space null spatial quaternionic curve in the semi-Euclidean space $\mathbb{R}_{\mathrm{v}}^{3}$. Then Frenet equations are

$$
l^{\prime}=u, n^{\prime}=\tau u, u^{\prime}=-\tau l-n,
$$

and

$$
l^{\prime}=u, n^{\prime}=-\tau u, u^{\prime}=\tau l-n,
$$

for $\mathbb{R}_{1}^{3}$, having Frenet frame $\{l, n, u\}$ and a single Cartan curvature $\tau$. Moreover, this null quaternionic Cartan frame $\{l, n, u\}$ satisfies the following conditions:

$$
h(l, l)=h(n, n)=h(l, u)=h(n, u)=0,
$$

and

$$
h(l, n)=h(u, u)=1 .
$$

Here, the quaternion product $\times$ is given by

$$
\begin{aligned}
& l \times n=-1-u, n \times l=-1+u, \\
& u \times n=n=-(n \times u), l \times u=l=-(u \times l) \\
& u \times u=-1, l \times l=n \times n=0,[10,11] .
\end{aligned}
$$

Definition 3.1.1. Let $(C, \bar{C})$ be a pair of framed null quaternionic curves in $\mathbb{R}_{\mathrm{v}}^{3}$, with distinguished paramerters $p$ and $\bar{p}$, respectively. This pair is said to be a null quaternionic Bertrand pair if their principal normal vector fields are linearly dependent (see [6] for null curves).

By above definition 3.1.1., there exist a functional relation $\bar{p}(p)=\bar{p}$ for a null quaternionic Bertrand pair $(C, \bar{C})$, such that $\bar{u}(\bar{p})=\mp u$ normal lines coincide at their respective points. To show that the null quaternionic Bertrand curves have been used in characterizing null quaternionic helices, let $C$ be a null quaternionic curve in $\mathbb{R}_{1}^{3}$, then its Cartan frame satisfies the following equations

$$
l^{\prime}=u, n^{\prime}=\tau u, u^{\prime}=-\tau l-n,
$$

and

$$
l^{\prime}=u, n^{\prime}=-\tau u, u^{\prime}=\tau l-n .
$$

Proposition 3.1.2. The distance $|\lambda|$ between corresponding points of a null quaternionic Bertrand curve and of its Bertrand mate (or Bertrand conjugate) is a constant.

Proof. Let $C(p)$ and $\bar{C}(\bar{p})$ be null quaternionic Bertrand curves, with respect to a special parameter $\bar{p}$ and suppose that $\{l, n, u\}$ and $\{\bar{l}, \bar{n}, \bar{u}\}$ are their quaternionic Cartan frames, respectively. Then we can write

$$
\bar{C}(\bar{p})=C(p)+\lambda(p) u(p),
$$

since the normal lines coincide. Suppose $p$ and $\bar{p}$ are the pseudo-arc parameters of $C$ and $\bar{C}$, respectively, then by taking derivative of Eq. (3.1) with respect to $p$ and using null quaternionic Cartan frame, we get $\frac{\mathrm{d} \bar{p}}{\mathrm{~d} p} \bar{l}=(1-\lambda \tau) l-\lambda n+\lambda^{\prime} u$. On the other hand, the condition $h(\bar{l}, u)=\frac{1}{2}(\bar{l} \times \alpha u+u \times \alpha \bar{l})=0$ holds for null quaternionic Bertrand curves, hence $\lambda^{\prime}=0$, we deduce that $\lambda$ is a nonzero constant. This means that the norm $\|\bar{C}-C\|$ is a constant.

Theorem 3.1.3. Null quaternionic Cartan curve in $\mathbb{R}_{1}^{3}$ is a null quaternionic Bertrand curve if and only if $\lambda$ is a non-zero constant. Moreover, $\bar{C}$ is a congruent to $C$. (Let $e_{1}$ is timelike in $\mathbb{R}_{1}^{3}$ ).

Proof. Suppose that $\bar{C}$ is a null quaternionic Bertrand mate for $C$. Then the previous theorem allows us to write

$$
\bar{l} \frac{\mathrm{d} \bar{p}}{\mathrm{~d} p}=(1-\lambda \tau) l-\lambda n,
$$

where $\lambda^{2}$ is the distance between the curves. Since $\bar{l}$ is null, $h(\bar{l}, \bar{l})=\bar{l} \times \alpha \bar{l}=0$ and $h(l, n)=1$ hold. We obtain $\lambda(1-\lambda \tau)=0$, so $\tau=\frac{1}{\lambda}=$ constant. Using this value of $\tau$ in Eq. (3.2), we obtain

$$
\bar{l} \frac{\mathrm{d} \bar{p}}{\mathrm{~d} p}=-\left(\frac{1}{\tau}\right) n .
$$

From Eq. (3.3), we have

$$
\bar{l}=-\frac{\mathrm{d} p}{\mathrm{~d} p} \frac{1}{\tau} n .
$$

and by differentiating Eq. (3.4) with respect to $p$ and using Cartan frame $\left(\bar{l}^{\prime}=\bar{u}\right.$ and $\left.n^{\prime}=\tau u\right)$, we get

$$
-u=\bar{u}\left(\frac{\mathrm{d} \bar{p}}{\mathrm{~d} p}\right)^{2}+\bar{l}\left(\frac{\mathrm{d}^{2} \bar{p}}{\mathrm{~d} p^{2}}\right) .
$$

Since $h(u, u)=u \times \alpha u=1$ and $\bar{u}=\mp u$ we get 
$\left(\frac{\mathrm{d} \bar{p}}{\mathrm{~d} p}\right)^{4}=1, \frac{\mathrm{d}^{2} \bar{p}}{\mathrm{~d} p^{2}}=0$ and $\bar{u}=-u$, so the Frenet frame of $C$ is $\left\{ \pm \lambda n, \pm \frac{1}{\lambda} l,-u\right\}$. Thus, $C$ has the same constant curvature $\tau=\frac{1}{\lambda} \neq 0$.

Conversely, let $C$ be a null quaternionic Cartan curve with constant curvature $\tau=\frac{1}{\lambda} \neq 0$. Then $\bar{C}(\bar{p})=$ $C(p)+\frac{1}{\tau}(p) u(p)$ is a null quaternionic Cartan curve with a pseudo arc parameter $p$ and framed by $\bar{l}=-\lambda n, \bar{n}=\frac{1}{\lambda} l$, $\bar{u}=-u$.

Consequently, the null quaternionic curve $\bar{C}$ is a null quaternionic Bertrand mate of $C$. The normal lines of the two curves coincide at corresponding points. Null quatenionic curves are parametrized by the pseudo-arc length parameter with Cartan first curvature always equal to 1 and the second curvature is always a non zero constant.

Example 3.1.4. Let us consider a null quaternionic helix curve in $\mathbb{R}_{1}^{3}$ defined by $C(p)=(p, \sin p, \cos p)$. Then Frenet frame is given by

$$
\begin{gathered}
F=\left\{C^{\prime}, N=\frac{1}{2}(1, \cos p,-\sin p),\right. \\
U=(0,-\sin p,-\cos p)\} .
\end{gathered}
$$

Since $h\left(n^{\prime}, u\right)=\tau$, we get $\tau=\frac{1}{2}$. From $\bar{C}(\bar{p})=C(p)+$ $\frac{1}{\tau}(p) u(p)$, we define $\bar{C}=C+2 u$ with $\bar{p}=p$. Hence, $\bar{C}=(p,-\sin p,-\cos p)$. Therefore, $\bar{C}$ is congruent to the null quaternionic curve $C$.

Proposition 3.1.5. Let $C(p)$ and $\bar{C}(\bar{p})$ be null quaternionic Bertrand curves, with respect to a special parameter $p$ and suppose that $\{l, n, u\}$ and $\{\bar{l}, \bar{n}, \bar{u}\}$ respectively. Suppose

$$
\bar{C}(\bar{p})=C(p)+\lambda_{1}(p) l(p)+\lambda_{2}(p) n(p)+\lambda_{3}(p) u(p),
$$

for some functions $\lambda_{1}, \lambda_{2}, \lambda_{3}$. Then, $\bar{C}$ is called an associated Cartan null quaternionic curve of $C$, with reference coordinate $\left(\lambda_{1}, \lambda_{2}, \lambda_{3}\right)$. By differentiating $\bar{C}$ we get

$$
\begin{aligned}
& \bar{l} \frac{\mathrm{d} \bar{p}}{\mathrm{~d} p}=\left(1+\lambda_{1}^{\prime}-\tau \lambda_{3}\right) l+\left(\lambda_{2}^{\prime}-\lambda_{3}\right) n \\
& \quad+\left(\lambda_{3}^{\prime}+\lambda_{1}+\lambda_{2} \tau\right) u .
\end{aligned}
$$

Since $L$ is null, $h(\bar{l}, \bar{l})=\bar{l} \times \alpha \bar{l}=0$ and $h(l, n)=1$ hold. We obtain $2\left(1+\lambda_{1}^{\prime}-\tau \lambda_{3}\right)\left(\lambda_{2}^{\prime}-\lambda_{3}\right)+\left(\lambda_{3}^{\prime}+\lambda_{1}+\lambda_{2} \tau\right)^{2}=0$ (see [5] for null curves).

\section{Theorem 3.1.6.}

(1) Let $C(p)$ be null quaternionic Cartan curve in $\mathbb{R}_{1}^{3}$, where $p$ is a special parameter. Assume that there exists a null quaternionic Cartan curve $\bar{C}(\bar{p})$ parameterized by special parameter $\bar{p}$, such that the binormal lines of $\bar{C}$ and $C$ coincide. Then, at the corresponding point, their Cartan curvatures coincide, that is, $\bar{\tau}(\bar{p})=\tau(p)$.

(2) Let $C(p)$ be null quaternionic Cartan curve in $\mathbb{R}_{1}^{3}$, where $p$ is a special parameter. There exists a null quaternionic Cartan curve $\bar{C}(\bar{p})$, parameterized by special parameter $\bar{p}$, such that the binormal lines of, and the respective curvatures of, $\bar{C}$ and $C$ coincide (see [5] for null curves).

Proof. (1) Assume a pair $(C(p), \bar{C}(\bar{p}))$ of null quaternionic Cartan curves with their special parameters $p$ and $\bar{p}$, repectively, such that their binormal lines coincide. Then, $C$ is parameterized as

$$
\bar{C}(\bar{p})=C(p)+v(p) n(p),
$$

for some function $v(p) \neq 0$ and some parameterization $\bar{p}(p)=\bar{p}$. Hence, $\bar{C}$ is an associated curve to $C$, with the reference coordinates $(0, v, 0)$. Thus without any loss of generality, we assume that

$$
\bar{n}(\bar{p})=\alpha_{1}(p) n(p),
$$

for some function $\alpha_{1}(p) \neq 0$. By differentiating Eq. (3.6) with respect to $p$, we get

$$
\bar{l} \frac{\mathrm{d} \bar{p}}{\mathrm{~d} p}=l+v^{\prime} n+v \tau u .
$$

In addition $h(\bar{l}, \bar{l})=\bar{l} \times \alpha \bar{l}=0$ and $h(l, n)=1$ yield

$$
2 v^{\prime}+(v \tau)^{2}=0 \text { and } \alpha+1=\frac{\mathrm{d} \bar{p}}{\mathrm{~d} p} \text {. }
$$

Thus, from Eq. (3.9), the function $v$ is completely determined by

$$
\frac{1}{v}=\frac{1}{2} \int \tau^{2}(p) \mathrm{d} p+c,
$$

for some constant $c$.

On the other hand, the condition

$$
\bar{l} \times \bar{n}=-1-\bar{u},
$$

holds for null quaternionic curves. Substituting (3.8) and (3.7) in (3.10), the principal normal $\bar{u}$ of $C$ can be expressed as

$$
\frac{\mathrm{d} p}{\mathrm{~d} \bar{p}}\left[\left(l+v^{\prime} n+v \tau u\right) \times \alpha_{1} n\right]=-1-\bar{u} .
$$

Here, using the quaternion product and making the necessary arrangements, we get

$$
\bar{u}=u-v \tau n \text {. }
$$

Differentiating Eq. (3.7) by $p$ and using Eq. (3.11) we obtain

$$
\bar{\tau} \frac{\mathrm{d} \bar{p}}{\mathrm{~d} p}(-u-\tau v n)=\alpha_{1}^{\prime} n-\alpha_{1} \tau u .
$$

Comparing both sides of above equation, we get $\bar{\tau}=\tau$, $\alpha_{1}^{\prime}=-\tau^{2} v \frac{\mathrm{d} \bar{p}}{\mathrm{~d} p}, \frac{\mathrm{d} \bar{p}}{\mathrm{~d} p}=a, \frac{\mathrm{d} a}{\mathrm{~d} p}=-\tau^{2} v a \Rightarrow a=a_{0} v^{2}$, $a_{0} \varepsilon \mathbb{R}^{+}$. Differentiating both sides of Eq. (3.11) and making necessary arrangements, we have

$$
\bar{\tau}=\tau,\left(\frac{\mathrm{d} \bar{p}}{\mathrm{~d} p}\right)^{2}=1+v \tau^{\prime} .
$$

So, the torsion at the corresponding points coincides. Since $\frac{\mathrm{d} \bar{p}}{\mathrm{~d} p}=a$, we get $a^{2}=1+v \tau^{\prime}$.

Proof. (2) For every null quaternionic Cartan curve $C(p)$, define a null quaternionic curve $\bar{C}(\bar{p})$ by $\bar{C}(\bar{p})=$ $C(p)+v(p) n(p), \frac{1}{v}=\frac{1}{2} \int \tau^{2}(p) \mathrm{d} p+c, c$ is a nonzero constant.

Since $\frac{\mathrm{d} \bar{p}}{\mathrm{~d} p}=a$ and $a=a_{0} v^{2}$, where $a_{0}$ is a nonzero constant, we get $\bar{p}(p)=a_{0} \int v^{2}(p) \mathrm{d} p$. Then $C$ is a Cartanframed null quaternionic curve, framed by

$$
\bar{l}=\frac{\mathrm{d} \bar{C}}{\mathrm{~d} p}, \bar{n}=\frac{\mathrm{d} \bar{p}}{\mathrm{~d} p} n, \bar{u}=-1-(\bar{l} \times \bar{n}) .
$$

Clearly, $C$ and $\bar{C}$ have common binormal directions. 


\section{Conclusions}

Let $C$ be a null quaternionic Cartan curve in $\mathbb{R}_{\mathrm{v}}^{3}$. Here, by using the similar idea of above theorems, we can obtain the following results

\begin{tabular}{|c|c|}
\hline Null quaternionic Frames in $\mathbb{R}_{\mathrm{v}}^{3}$ & Results \\
\hline $\begin{array}{l}(\mathbf{I}-\mathbf{a}) l^{\prime}=u, n^{\prime}=\tau u, u^{\prime}=-\tau l-n \\
e_{1} \text { is a timelike vector. }\end{array}$ & $\begin{array}{l}\text { Since } \bar{C}(\bar{p})=C(p)+\lambda(p) u(p), \text { we get } \tau=\frac{1}{\lambda} \neq 0 . \\
\text { From } \bar{C}(\bar{p})=C(p)+v(p) N(p), \\
\text { we get } 2 v^{\prime}+(v \tau)^{2}=0, \bar{u}=u-v \tau n \text { and } \bar{\tau}=\tau .\end{array}$ \\
\hline $\begin{array}{l}(\mathbf{I}-\mathbf{b}) l^{\prime}=u, n^{\prime}=-\tau u, u^{\prime}=\tau l-n \\
e_{1} \text { is a timelike vector. }\end{array}$ & $\begin{array}{l}\bar{C}(\bar{p})=C(p)+\lambda(p) u(p), \text { we get } \tau=-\frac{1}{\lambda} \neq 0 \\
\text { From } \bar{C}(\bar{p})=C(p)+v(p) N(p) \\
\text { we get } 2 v^{\prime}+(v \tau)^{2}=0, \bar{u}=u+v \tau n \text { and } \bar{\tau}=\tau .\end{array}$ \\
\hline $\begin{array}{l}(\mathbf{I I}-\mathbf{a}) l^{\prime}=u, n^{\prime}=-\tau u, u^{\prime}=-\tau l+n \\
e_{2} \text { is a timelike vector. }\end{array}$ & $\begin{array}{l}\text { From } \bar{C}(\bar{p})=C(p)+\lambda(p) u(p), \text { we get } \tau=\frac{1}{\lambda} \neq 0 . \\
\text { Since } \bar{C}(\bar{p})=C(p)+v(p) n(p) \\
\text { we have }-2 v^{\prime}+(v \tau)^{2}=0, \bar{u}=u-v \tau n \text { and } \bar{\tau}=\tau .\end{array}$ \\
\hline $\begin{array}{l}(\mathbf{I I}-\mathbf{b}) l^{\prime}=u, n^{\prime}=\tau u u^{\prime}=\tau l+n \\
e_{2} \text { is a timelike vector. }\end{array}$ & $\begin{array}{l}\text { From } \bar{C}(\bar{p})=C(p)+\lambda(p) u(p), \text { we get } \tau=-\frac{1}{\lambda} \neq 0 . \\
\text { Since } \bar{C}(\bar{p})=C(p)+v(p) n(p), \\
\text { we have }-2 v^{\prime}+(v \tau)^{2}=0, \bar{u}=u+v \tau n \text { and } \bar{\tau}=\tau .\end{array}$ \\
\hline $\begin{array}{l}(\mathbf{I I I}-\mathbf{a}) l^{\prime}=u, n^{\prime}=-\tau u, u^{\prime}=-\tau l+n \\
e_{1} \text { and } e_{2} \text { are timelike vectors. }\end{array}$ & $\begin{array}{l}\text { Since } \bar{C}(\bar{p})=C(p)+\lambda(p) u(p), \text { we get } \tau=\frac{1}{\lambda} \neq 0 . \\
\text { From } \bar{C}(\bar{p})=C(p)+v(p) n(p), \\
\text { we have } 2 v^{\prime}-(v \tau)^{2}=0, \bar{u}=u-v \tau n \text { and } \bar{\tau}=\tau .\end{array}$ \\
\hline $\begin{array}{l}(\mathbf{I I I}-\mathbf{b}) l^{\prime}=u, n^{\prime}=\tau u, u^{\prime}=\tau l+n \\
e_{1} \text { and } e_{2} \text { are timelike vectors. }\end{array}$ & $\begin{array}{l}\text { Since } \bar{C}(\bar{p})=C(p)+\lambda(p) u(p), \text { we get } \tau=-\frac{1}{\lambda} \neq 0 \text {. } \\
\text { From } \bar{C}(\bar{p})=C(p)+v(p) n(p), \\
\text { we have } 2 v^{\prime}-(v \tau)^{2}=0, \bar{u}=u+v \tau n \text { and } \bar{\tau}=\tau .\end{array}$ \\
\hline $\begin{array}{l}(\mathbf{I V}-\mathbf{a}) l^{\prime}=u, n^{\prime}=\tau u, u^{\prime}=-\tau l-n \\
e_{2} \text { and } e_{3} \text { are timelike vectors. }\end{array}$ & $\begin{array}{l}\text { Since } \bar{C}(\bar{p})=C(p)+\lambda(p) u(p), \text { we get } \tau=\frac{1}{\lambda} \neq 0 . \\
\text { From } \bar{C}(p)=C(\bar{p})+v(p) n(p), \\
\text { we have } 2 v^{\prime}+(v \tau)^{2}=0, \bar{u}=u-v \tau n \text { and } \bar{\tau}=\tau\end{array}$ \\
\hline $\begin{array}{l}(\mathbf{I V}-\mathbf{b}) l^{\prime}=u, n^{\prime}=-\tau u, u^{\prime}=\tau l-n \\
e_{2} \text { and } e_{3} \text { are timelike vectors. }\end{array}$ & $\begin{array}{l}\text { Since } \bar{C}(\bar{p})=C(p)+\lambda(p) u(p), \text { we get } \tau=-\frac{1}{\lambda} \neq 0 \text {. } \\
\text { From } \bar{C}(\bar{p})=C(p)+v(p) n(p), \\
\text { we have } 2 v^{\prime}+(v \tau)^{2}=0, \bar{u}=u+v \tau n \text { and } \bar{\tau}=\tau .\end{array}$ \\
\hline
\end{tabular}

\section{References}

[1] K. Bharathi, M. Nagaraj, Indian J. Pure Appl. Math. 18, 507 (1987).

[2] W.B. Bonnor, Tensor N.S. 20, 229 (1969).

[3] A.C. Çöken, A. Tuna, Appl. Math. Comput. 155 , 373 (2004).

[4] K.L. Duggal, A. Bejancu, Lightlike submanifolds of semi-Riemannian manifolds and applications, Kluwer Academic, 1996.

[5] K.L. Duggal, D.H. Jin, Null curves and hypersurfaces of semi-Riemannian manifolds, World Scientific Publishing, 2007.
[6] A. Fernandez, A. Gimenez, P. Lucas, Int. J. Mod. Phys. 16, 4845 (2001).

[7] K. Honda, J. Inoguchi, Differential Geom.-Dynam. Syst. 5, 31 (2003).

[8] J. Inoguchi, S. Lee, Int. Electronic J. Geom. 1, 40 (2008).

[9] O. Keçilioğlu, K. İlarslan, Bull. Math. Anal. Applicat 5, 27 (2013).

[10] A. Tuna Aksoy, Ph.D Thesis, Süleyman Demirel University, 2014.

[11] A. Tuna Aksoy, A.C. Çöken, Acta Phys. Pol. A 128, B-286 (2015). 